\title{
A Novel Triterpene Saponin from Gypsophila capillaris
}

\author{
M. Hani A. Elgamal, Hesham S. M. Soliman \\ National Research Centre, Laboratory of Natural Products, Dokki-Cairo, Egypt \\ Helmut Duddeck* \\ Universität Hannover, Institut für Organische Chemie, Schneiderberg 1 B, \\ D-30167 Hannover, Bundesrepublik Deutschland \\ Bozhana Mikhova \\ Bulgarian Academy of Sciences, Institute of Organic Chemistry, Sofia, Bulgaria \\ Martin Gartmann \\ Ruhr-Universität Bochum, Fakultät für Chemie, Postfach 102148 , \\ D-44780 Bochum, Bundesrepublik Deutschland \\ Z. Naturforsch. 50b, 563-567 (1995); received October 28, 1994 \\ Gypsophila capillaris, Caryophyllaceae, Quillaic Acid, Glucose, Galactose
}

A novel C-28 tetraglycoside of quillaic acid (1) has been isolated from Gypsophila capillaris. The structure was elucidated by $1 \mathrm{D}$ NMR (NOE difference, DEPT, selective ${ }^{13} \mathrm{C}\left\{{ }^{1} \mathrm{H}\right\}$ INEPT), 2 D NMR $\left({ }^{1} \mathrm{H},{ }^{1} \mathrm{H}\right.$ and ${ }^{1} \mathrm{H},{ }^{13} \mathrm{C}$ COSY, ${ }^{1} \mathrm{H},{ }^{1} \mathrm{H},{ }^{1} \mathrm{H}$ RELAY, ROESY and TOCSY) and other spectroscopic and chromatographic evidences. Conformational dynamics within the tetrasaccharide part were estimated from NOE responses and ROESY peaks.

\section{Introduction}

Gypsophila capillaris (Forssk.) has been collected in the eastern Egyptian desert between Cairo and Suez. Recently, we communicated six new triterpene glycosides isolated from this plant $[1,2]$. Due to unfortunate signal overlaps the NMR spectra of a seventh saponin (1) could not be interpreted unambiguously on the basis of the methods described previously [1,2] so that we had to apply further experiments, such as 2D TOCSY. In this paper we wish to report the structure of $\mathbf{1}$, quillaic acid ( $3 \beta, 16 \alpha$-dihydroxyolean-12-en23-oxo-28-oic acid) 28-O-[ $\beta$-D-glucopyranosyl$(1 \rightarrow 2)][\beta$-D-glucopyranosyl $(1 \rightarrow 2)$ - $\beta$-D-galactopyranosyl $(1 \rightarrow 6)] \beta$-D-glucopyranoside, along with some comments on the conformational behaviour.

\section{Results and Discussion}

Structural determinations are mainly based on the NMR spectral assignments from homo- and heteronuclear COSY, ${ }^{1} \mathrm{H},{ }^{1} \mathrm{H},{ }^{1} \mathrm{H}$ RELAY experiments, homonuclear NOE difference, ROESY and TOCSY experiments [3]. They are confirmed by

\footnotetext{
* Reprint requests to Prof. Dr. H. Duddeck.
}

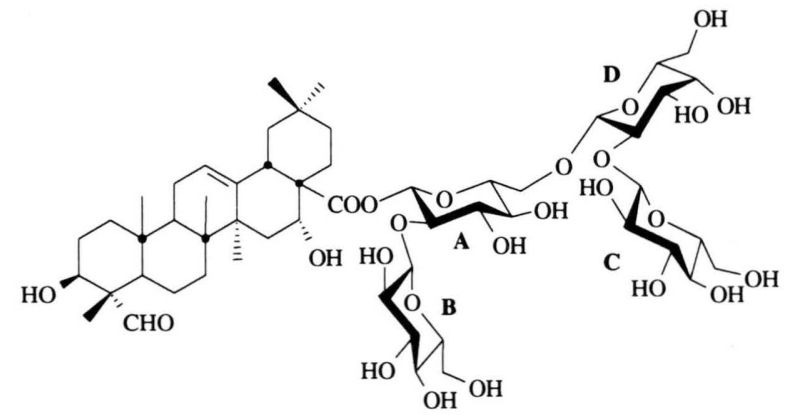

Scheme 1. Structure of $\mathbf{1}$.

$$
\begin{gathered}
\mathrm{R}-\mathrm{C}^{28}(=\mathrm{O})-\underset{\mid}{\mathrm{O}}-\mathrm{glc}[\mathbf{A}]-(6 \leftarrow 1)-\operatorname{gal}[\mathbf{D}]-(2 \leftarrow 1)-\operatorname{glc}[\mathbf{C}] \\
(2 \leftarrow 1)-\operatorname{glc}[\mathbf{B}] .
\end{gathered}
$$

All monosaccharides are $\beta$-D-pyranoses; the orientation of the monosaccharide subunits is arbitrary in this scheme. For the conformational behavior see Scheme 2 and text.

the molecular ions and fragmentation peaks in the mass spectra.

After hydrolysis of $\mathbf{1}$ the aglycone quillaic acid was easily identified by comparison of its ${ }^{13} \mathrm{C}$ NMR spectrum with literature data [4] and by chromatographic comparison with an authentic sample. The ${ }^{13} \mathrm{C}$ data found are very close to the

0932-0776/95/0400-0563\$06.00 (c) 1995 Verlag der Zeitschrift für Naturforschung. All rights reserved. 
reported values so that we refrain from including them in Table I except some diagnostic signals (Table I).

Acidic hydrolysis of $\mathbf{1}$ afforded only glucose and galactose as determined by GC (persilylated) and TLC comparison with authentic sugars. The C-3 and H-3 NMR signals of $\mathbf{1}$ showed chemical shifts very close to those observed in the free aglycone so that we can assure that the saccharide part of the molecule is attached exclusively to the C-28 carboxylic acid group. This is supported by the finding that alkaline hydrolysis of $\mathbf{1}$ afforded the aglycones without any further sugars residues.

The procedure of assigning the ${ }^{1} \mathrm{H}$ and ${ }^{13} \mathrm{C}$ signals, determining the stereochemistry of the monosaccharides $(\alpha$ or $\beta$ ) and establishing the interglycosidic linkages by using the above mentioned NMR techniques has been described by us previously $[1,2]$. In the case of $\mathbf{1}$, however, we encountered some serious signal overlaps which did not allow an unambiguous structural determination. Thus, we recorded a TOCSY spectrum with a long mixing time $(200 \mathrm{~ms})$ in order to find a safe assignment of the ${ }^{1} \mathrm{H}$ signals in each mono-

Table I. ${ }^{1} \mathrm{H}$ and ${ }^{13} \mathrm{C}$ chemical shifts of the saccharide part of 1; selected diagnostic aglycone signals are included ${ }^{\mathrm{a}}$.

\begin{tabular}{lllll}
\hline & $\begin{array}{l}\text { Sugar A } \\
\text { (glc) }\end{array}$ & $\begin{array}{l}\text { Sugar B } \\
\text { (glc) }\end{array}$ & $\begin{array}{l}\text { Sugar C } \\
\text { (glc) }\end{array}$ & $\begin{array}{l}\text { Sugar D } \\
\text { (gal) }\end{array}$ \\
\hline H-1 & 6.09 & 5.74 & 5.19 & 4.90 \\
H-2 & 4.65 & 4.01 & 3.99 & 4.02 \\
H-3 & 4.22 & 4.18 & 4.09 & 4.18 \\
H-4 & 4.58 & 4.02 & 4.17 & 4.16 \\
H-5 & 3.93 & 4.02 & 3.79 & 3.74 \\
H-6/6'b & $4.50 / 4.25$ & $4.66 / 4.33$ & $4.59 / 4.42$ & $4.35 / 4.28$ \\
C-1 & 93.6 & 104.1 & 106.2 & 102.8 \\
C-2 & 77.6 & 72.8 & 76.3 & 84.5 \\
C-3 & 78.7 & 78.4 & 78.2 & 78.4 \\
C-4 & 69.9 & 71.8 & 70.8 & 71.1 \\
C-5 & 77.3 & 78.4 & 78.6 & 78.4 \\
C-6 & 68.9 & 63.9 & 62.2 & 62.2
\end{tabular}

Aglycone:

\begin{tabular}{lr} 
H-3 & 4.00 \\
H-16 & 4.01 \\
C-3 & 71.8 \\
C-16 & 75.9 \\
C-28 & 176.9 \\
\hline
\end{tabular}

a In pyridine $\mathrm{d}_{5}$; relative to the lowest-frequency ${ }^{1} \mathrm{H}$ signal of pyridine- $\mathrm{d}_{4}(\delta=7.19)$ and to the lowest-frequency ${ }^{13} \mathrm{C}$ signal of pyridine- $\mathrm{d}_{5}(\delta=123.8)$, respectively; ${ }^{\mathrm{b}}$ the atoms $\mathrm{H}-6$ and $\mathrm{H}-\mathrm{6}^{\prime}$ were not stereo-differentiated.

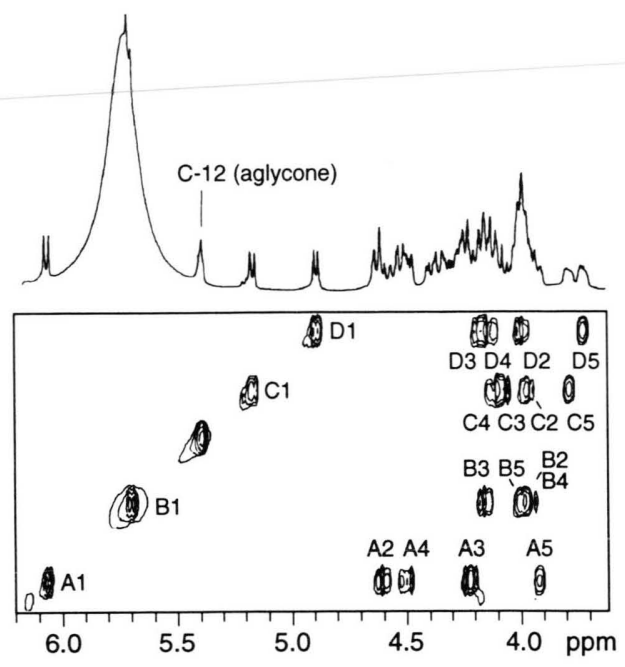

Fig. 1. TOCSY spectrum of $\mathbf{1}$ (anomeric region) demonstrating the ${ }^{1} \mathrm{H}$ signal assignment within the monosaccharide subunits $\mathbf{A}$ to $\mathbf{D}$.

saccharide subunit. Fig. 1 shows a section of the TOCSY spectrum in the region of the anomeric ${ }^{1} \mathrm{H}$ signals. The cross-peaks reveal the exact positions of the signals of $\mathrm{H}-2$ to $\mathrm{H}-5$ within each monosaccharide subunit (A to D). Cross-peaks of the H-6 signals did not appear. However, their position could be determined unambiguously by the ${ }^{1} \mathrm{H},{ }^{13} \mathrm{C}$ COSY and by correlating them with the respective $\mathrm{H}-5$ signals in the ${ }^{1} \mathrm{H},{ }^{1} \mathrm{H}$ COSY and TOCSY spectra.

The interglycosidic linkages were established by selective INEPT experiments where the anomeric hydrogens were irradiated and responses from those ${ }^{13} \mathrm{C}$ nuclei appeared which carry the monosaccharide subunit with the irradiated proton $\left[{ }^{1} \mathrm{H}(\mathrm{A} 1) \rightarrow{ }^{13} \mathrm{C}-28\right.$ (aglycone); ${ }^{1} \mathrm{H}(\mathrm{B} 1) \rightarrow{ }^{13} \mathrm{C}(\mathrm{A} 2)$; $\left.{ }^{1} \mathrm{H}(\mathrm{C} 1) \rightarrow{ }^{13} \mathrm{C}(\mathrm{D} 2) ;{ }^{1} \mathrm{H}(\mathrm{D} 1) \rightarrow{ }^{13} \mathrm{C}(\mathrm{A} 6)\right]$.

It has been mentioned before $[1,2]$ that the NOE difference spectra of the saccharide part gave negative signals due to the motional behavior of the molecule (Fig. 2) [5]. Nevertheless, the NOE responses on irradiation of the anomeric protons of $\mathbf{1}$ were of sufficient intensity to confirm the above mentioned interglycosidic linkages. Moreover, we could clearly see the respective H-3 signals, which allowed to differentiate between glucose (triplet) and galactose (doublet), and the respective $\mathrm{H}-5$ signals, which afforded the connectivities to the $\mathrm{H}-6 / \mathrm{H}-6$ ' protons (see 


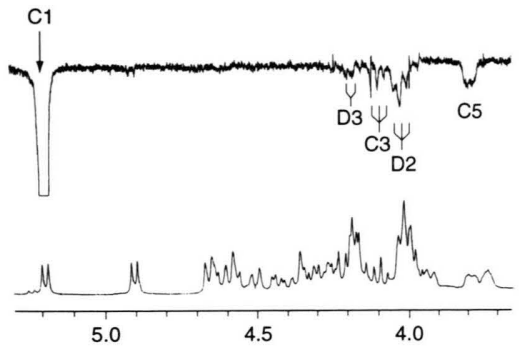

Fig. 2. NOE difference spectrum of $\mathbf{1}$; irradiation on the position of the anomeric ${ }^{1} \mathrm{H}$ signal of monosaccharide $\mathbf{C}$ (C1, $\delta=4.90)$; the same responses occurred as crosspeaks in the ROESY spectrum.

above). Since, however, we found some additional signals in the NOE difference spectra we recorded the ROESY spectrum of $\mathbf{1}$ and found cross-peaks at the same ${ }^{1} \mathrm{H}$ positions. There is even a qualitative correspondence of the signal magnitudes in those two spectra. These evidences allowed to give some preliminary comments of the conformational behavior of the saponin 1; a more detailed analysis by molecular modelling methods is currently under investigation.

Relative orientation of $\mathbf{A}$ and $\mathbf{B}$ (Scheme 2, top): Irradiation on the anomeric proton of $\mathbf{B}$ (B 1) gave a strong response of $\mathrm{A} 2$ and a weak one of $\mathrm{A} 3$ (dashed arrows), but A 1 does not respond. Thus, under the assumption that the exo-anomeric effect (gauche-orientation of the torsion angle $\mathrm{O}-\mathrm{B} 1-$ $\mathrm{O}-\mathrm{A} 2$ ) [6] is operating, the structure depicted in Scheme 2 (top) represents a conformation family with close atoms B 1 and A 2. A ca. $60^{\circ}$ anti-clockwise torsion around the bond between $\mathrm{B} 1$ and the exocyclic oxygen leads to an approach of B 1 and A 3 explaining the observed weak NOE response. Further torsion around larger angles, however, does not take place so that B1 and A 1 cannot come close to each other in space.

Relative orientation of $\mathbf{A}$ and $\mathbf{D}$ (Scheme 2, middle): Irradiation on the anomeric proton of D (D1) gave NOE responses of both A 6 and A 6' with comparable intensities. Thus, we conclude that A 5 and D 1 are antiperiplanar. Any torsional angle A 5-A 6-O-D 1 smaller than $\left|120^{\circ}\right|$ can be excluded because there is no noticeable A 5-D 1 contact. Such conformation is prevented by steric interaction between the monosaccharides $\mathbf{C}$ and $\mathbf{A}$.

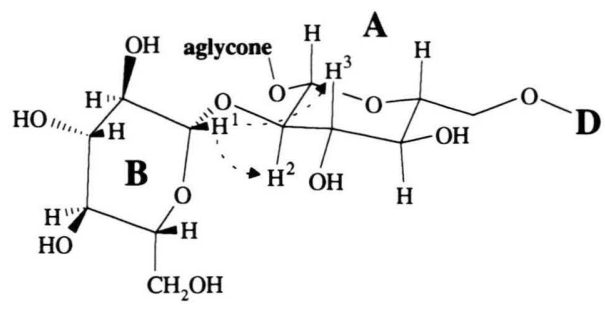

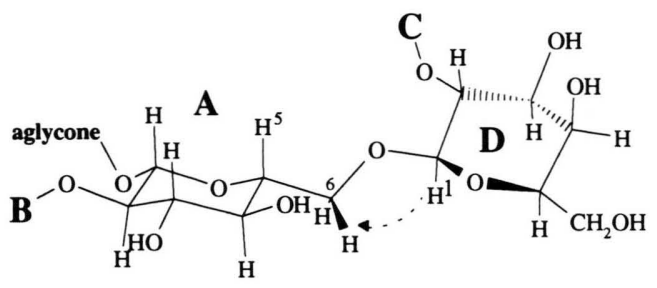

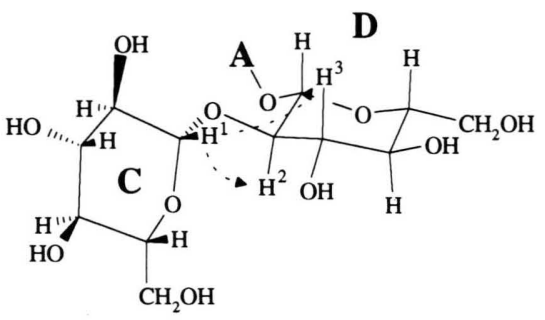

Scheme 2. Estimated conformational preference within the tetrasaccharide part of $\mathbf{1}$; arrows indicate NOE contacts; the letter $\mathbf{A}$ to $\mathbf{D}$ indicate the monosaccharide subunits (compare Scheme 1).

Relative orientation of $\mathbf{C}$ and $\mathbf{D}$ (Scheme 2, bottom): Irradiation on the anomeric proton of $\mathbf{C}$ (C1) gave NOE responses analogous to the $\mathbf{A} / \mathbf{B}$ connectivity so that a similar conformational behavior can be assumed.

We expect analogous dynamics for the six related saponins described by us previously $[1,2]$.

The positive-ion FAB mass spectra gave the expected peaks for the aglycone, mono-, di-, tri- and tetraglycoside ions (see Experimental part).

It is interesting to note that the structure of $\mathbf{1}$ is closely related to the quillaic acid triglycoside published by us before where sugar $\mathbf{C}$ is missing (compound $\mathbf{2}$ in ref. [1]). ${ }^{1} \mathrm{H}$ chemical shifts of the monosaccharide subunit $\mathbf{D}$ do not give any safe indication of the attachment of sugar $\mathbf{C}$ although both compounds have been recorded in the same solvent and in comparable concentrations. On the 
other hand, glucosidation shifts can be determined for two ${ }^{13} \mathrm{C}$ resonances of sugar $\mathbf{D}: 8.8 \mathrm{ppm}$ deshielding for $\mathrm{C}-2$ ( $\beta$-effect) and 2.5 shielding for $\mathrm{C}$-1 $(\gamma$-effect). There is, however, no $\gamma$-effect on C-3.

\section{Experimental}

General: Melting points were uncorrected. IR spectra were taken on a Bruker IFS-25 in $\mathrm{KBr} .{ }^{1} \mathrm{H}$ and ${ }^{13} \mathrm{C}$ NMR spectra were recorded in pyridine$\mathrm{d}_{5}$ using Bruker AM-400 $\left({ }^{1} \mathrm{H}: 400.1 \mathrm{MHz} ;{ }^{13} \mathrm{C}\right.$ : $100.6 \mathrm{MHz})$ and AM-300 $\left({ }^{1} \mathrm{H}: 300.1 \mathrm{MHz} ;{ }^{13} \mathrm{C}\right.$ : $75.4 \mathrm{MHz}$ ) spectrometers. Standard Bruker software has been applied for most of the multipulse NMR experiments. Duration of presaturation in the NOE difference experiments was $1.5 \mathrm{~s}$, relaxation delay $5 \mathrm{~s}$. Mixing times were $200 \mathrm{~ms}$ and trim pulses were $2.5 \mathrm{~ms}$ in both the ROESY and the TOCSY experiment; relaxation delays were $2 \mathrm{~s}$. The Bruker standard ROESY and TOCSY pulse sequences were modified by applying $z$-filtration [7]. FAB mass spectra were obtained with a VG Autospec (in lactic acid). Column chromatography was performed using silica gel 60 (140-270 mesh); TLC with silica gel $60 \mathrm{~F}_{254}$ plates (Merck); GC with a column SE-54 (Macherey \& Nagel) and FID detector, carrier gas was $\mathrm{N}_{2}$. Elemental analysis have not yet been performed in order to save the material for biological studies.

Gypsophila capillaris (Forssk.) growing in the eastern Egyptian desert between Cairo and Suez has been collected and identified by Prof. L. Bolous (National Research Centre, NRC), Cairo. A voucher specimen is kept in the herbarium of the NRC. The powder form of the whole plant (aerial parts and roots, $2.5 \mathrm{~kg}$ ) was extracted with methanol, and the solvent was removed under reduced pressure. The methanolic extract was then dissolved in water and extracted with ether. The defatted aqueous layer was exhaustively extracted with $n$-butanol, and the solvent was removed under reduced pressure. The $n$-butanol extract ( $35 \mathrm{~g})$ was chromatographed on a silica gel column and eluted by a mixture of chloroform, methanol and water $(100: 10: 1)$. For further elution the chloroform content was reduced stepwise until a mixture composition of 10:10:1 was reached. Finally, pure methanol was used.

The fraction collected with a solvent mixture $(40: 10: 1)$ gave $1.4 \mathrm{~g}$ of a crude product from which we have isolated two novel saponins as de- scribed previously $[1,2]$. When the composition of the solvent mixture reached $(30: 10: 1) 0.9 \mathrm{~g}$ of a crude product was eluted providing another saponin [2]. A further fraction using the same polarity $(30: 10: 1)$ gave $1.2 \mathrm{~g}$ of a crude product; TLC of this fraction using chloroform, methanol and water $(18: 11: 2.7)$ showed one major spot $\left(R_{f}=\right.$ $0.35)$. Its purification was achieved by mediumpressure reversed-phase chromatography (RP-8, $48 \%$ methanol) followed by filtration over Sephadex LH-20 (Pharmacia, Sweden) using methanol to isolate $140 \mathrm{mg}$ of $\mathbf{1}$ in pure form.

\section{Alkaline hydrolysis of $\mathbf{1}$}

$20 \mathrm{mg}$ of aqueous methanolic solution of $\mathbf{1}$ were refluxed with $5 \% \mathrm{KOH}$ for $3 \mathrm{~h}$. The solutions were neutralized with diluted $\mathrm{HCl}$ and extracted with $n$-butanol, saturated with water. The $n$-butanol extract contained quillaic acid; $R_{f}=0.3$ using chloroform and methanol $(11: 1)$ as solvent system.

\section{Acidic hydrolysis of $\mathbf{1}$}

The aqueous methanolic solution of $\mathbf{1}(15 \mathrm{mg})$ was treated with $9 \% \mathrm{HCl}$ under reflux for $6 \mathrm{~h}$. Working up as usual gave quillaic acid. The aqueous layer was neutralized with barium carbonate for the sugar test.

(3 $\beta, 16 \alpha$-Dihydroxyolean-12-en-23-oxo-28-oic acid) 28 -O-[ $\beta$-D-glucopyranosyl $(1 \rightarrow 2)][\beta$-D-glucopyranosyl $(1 \rightarrow 2)$ - $\beta$-D-galactopyranosyl $(1 \rightarrow 6)]$ $\beta$-D-glucopyranoside (1)

M.p. $220-222^{\circ} \mathrm{C} .-[\alpha]_{\mathrm{D}}^{20}=+9.9(10.6 \mathrm{mg} / \mathrm{ml}$ $\mathrm{MeOH}) .-\mathrm{IR}(\mathrm{KBr}) v=3402(\mathrm{OH}), 1724(\mathrm{C}=\mathrm{O})$, $1078(\mathrm{C}-\mathrm{O}) \mathrm{cm}^{-1} .-{ }^{1} \mathrm{H}$ and ${ }^{13} \mathrm{C}$ NMR (pyridine$\mathrm{d}_{5}$ ) see Table I. - FAB MS $\mathrm{m} / \mathrm{z}=487$ [aglycone + $\mathrm{H}]^{+} ; 649$ [aglycone +1 hexose $\left.+\mathrm{H}\right]^{+} ; 811$ [aglycone +2 hexoses $+\mathrm{H}]^{+} ; 973$ [aglycone +3 hexoses + $\mathrm{H}]^{+} ; 1135$ [aglycone +4 hexoses $\left.+\mathrm{H}\right]^{+}$(molecular weight: $\mathrm{C}_{54} \mathrm{H}_{86} \mathrm{O}_{25}$ ).

\section{Acknowledgements}

H. S. M. Soliman thanks the German Academic Exchange Service (DAAD) for a scholarship in the frame of the channel program between the governments of Germany and Egypt. B. Mikhova thanks the Deutsche Forschungsgemeinschaft for a fellowship at Hannover University in 1994. Financial support was provided by Deutsche Forschungsgemeinschaft, Gesellschaft für Technische Zusammenarbeit and Fonds der Chemischen Industrie. 
[1] M. H. A. Elgamal, H. S. M. Soliman, M. H. S. Karawya, H. Duddeck, Nat. Prod. Lett. 4, 217 (1994).

[2] M. H. A. Elgamal, H. S. M. Soliman, M. H. S. Karawya, H. Duddeck, B. Mikhova, Phytochemistry, in press.

[3] a) W. R. Croasmun, R. M. K. Carlson (eds): TwoDimensional NMR Spectroscopy, Applications for Chemists and Biochemists, 2nd ed., VCH Publishers, New York (1994);

b) G. E. Martin, A. S. Zektzer, Two-Dimensional Methods for Establishing Molecular Connectivity, VCH Publishers, New York (1988); c) all NMR experiments were performed by using standard Bruker software.

[4] K. Tori, S. Seo, A. Shimaoka, Y. Tomita, Tetrahedron Lett. 1974, 4227.

[5] D. Neuhaus, M. P. Williamson, The Nuclear Overhauser Effect in Structural and Conformational Analysis, pp. $89 \mathrm{ff}$., VCH, New York (1989).

[6] A. J. Kirby, The Anomeric Effect and Related Stereoelectronic Effects at Oxygen, Springer, Heidelberg (1983).

[7] M. Rance, J. Magn. Reson. 74, 557 (1987). 